\title{
How Anthropomorphism Affects Empathy Toward Robots
}

\author{
Laurel D. Riek*, Tal-Chen Rabinowitch ${ }^{\dagger}$, Bhismadev Chakrabarti ${ }^{\ddagger}$ \\ \& Peter Robinson*
}

\author{
*Computer Laboratory \\ University of Cambridge \\ Cambridge, UK \\ $\{\mid r 314, p r 10\} @ c a m . a c . u k$
}

\author{
${ }^{\dagger}$ Centre for Music and Science \\ University of Cambridge \\ Cambridge, UK \\ tcnr2@cam.ac.uk
}

\author{
${ }^{\ddagger}$ Autism Research Centre \\ University of Cambridge \\ Cambridge, UK \\ bc249@cam.ac.uk
}

\begin{abstract}
A long-standing question within the robotics community is about the degree of human-likeness robots ought to have when interacting with humans. We explore an unexamined aspect of this problem: how people empathize with robots along the anthropomorphic spectrum. We conducted an experiment that measured how people empathized with robots shown to be experiencing mistreatment by humans. Our results indicate that people empathize more strongly with more human-looking robots and less with mechanicallooking robots.
\end{abstract}

Categories and Subject Descriptors: I.2.9 [Artificial Intelligence]: Robotics-Commercial robots and applications; J.4 [Computer Applications]: Social and Behavioral SciencePsychology, Sociology

General Terms: Experimentation, Human Factors

\section{INTRODUCTION}

A continuing question within the robotics community concerns the degree of human-likeness robots ought to have when interacting with humans. This question is often framed within the context of the Uncanny Valley effect, which is a theory proposed by Mori that posits as robots become more humanlike they become more familiar (and thus more likeable) until the mismatch between their form, interactivity, and motion quality elicits a sense of unease [3]. This notion seems plausible given the idea of Simulation Theory, an established theory in psychology that suggests that the way in which we understand the minds of others is through "simulating" another's situation (i.e. putting ourselves in their shoes) in order to understand their mental state/emotion.

If such a simulative system does exist, it follows that it should be easier to empathize with the emotions and mental states of an agent that appears similar to us than with one that does not. A large number of social psychology studies

Permission to make digital or hard copies of all or part of this work for personal or classroom use is granted without fee provided that copies are not made or distributed for profit or commercial advantage and that copies bear this notice and the full citation on the first page. To copy otherwise, to republish, to post on servers or to redistribute to lists, requires prior specific permission and/or a fee.

HRI'09, March 11-13, 2009, La Jolla, California, USA.

Copyright 2009 ACM 978-1-60558-404-1/09/03 ...\$5.00. support this and point to the fact that in-group bias and consequent referential treatment can be triggered by markers of physical similarity (e.g. skin color) [6]. In addition, evidence from developmental psychology suggests that children from birth use a simulation metric ('like me') as a building block from birth to detect others' emotions and mental states [5].

In robotics, several researchers have tested how people view robots of varying degrees of human-likeness. Goetz et al. showed people prefer more humanlike robots for jobs requiring more sociability [1]. Hinds et al. showed that when collaborating with robots of varying degrees of human likeness people took less credit for work done and less personal responsibility as robots were more humanlike [2]. Krach et al. showed a linear relationship between degree of anthropomorphization and cortical activation in brain areas related to how we process other minds [4].

One dimension of the human-likeness problem that remains unexplored is the degree to which people empathize with robots along the anthropomorphic spectrum. How does the degree of human-likeness affect empathy? Would observing robots in distress evoke a sense of charity? On the basis of Simulation Theory, as well as prior results reported in the robotics literature, we predicted that there will be an anthropomorphic gradient in the degree to which people empathize with robots, i.e. the more humanoid a robot looks, the more people will empathize with it.

Thus, we designed an experiment to test the hypothesis that people will be more empathetic toward human-like robots and less empathetic toward mechanical-like robots. We examined how people empathized with a variety of robots shown to be experiencing mistreatment by humans.

\section{METHODOLOGY}

We created a within-subjects, web-based survey in which people watched 30 -second film clips featuring five protagonists of varying degrees of human-likeness (See Fig. 2). Roomba is a disc-like, wheeled robotic vacuum cleaner that can beep several tones. AUR is an LED robotic lamp with 5 degrees-of-freedom; it is silent but can use its light to convey a range of colors and intensities. Andrew is an adult-sized humanoid with full range of movement, limited facial expressitivity, and has a slightly mechanical sounding voice. Alicia is an adult-sized android with a full range of movement, a fully human appearance, and has a mostly-human sounding voice. Anton is a human boy.

For each protagonist subjects viewed one emotionally evocative and one neutral clip. There were ten clips in all. In 


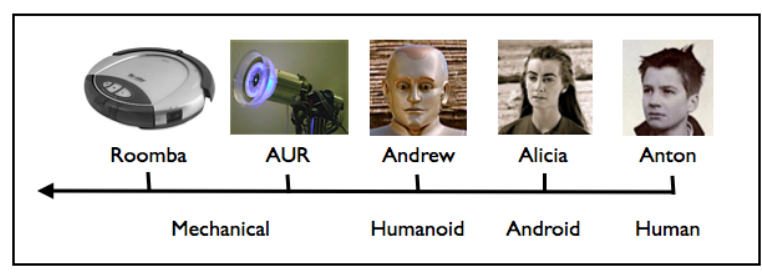

Figure 1: The protagonists used in the experiment.

the emotionally evocative clips, humans acted exceptionally cruel to the protagonist, such as shouting at them, pushing them, or ordering them to do embarrassing things. In the neutral clips, the protagonist was shown doing something mundane such as cleaning the cellar or setting the table.

For each clip respondents received the following set of screens. First, a black and white picture of the protagonist. Then, the 30-second clip featuring the protagonist. Last, subjects rated on a Likert scale how sorry they felt for the protagonist ( $1=$ not at all, $6=$ extremely). This single-question approach allowed subjects to express a more "raw" empathy response to the stimulus because they didn't have to give a lot of thought to their reply.

Immediately following the film clip section, subjects were shown pictures of the four robots with the following question: "Imagine there's been an earthquake and you can only save one of the robot protagonists. Which one would you save?" This question is similar to instruments used in other empathy studies regarding the notion of charity. Such phrasing targets the pro-social behavioral component of empathy (i.e. the component of empathy that leads to "helping" behavior toward others in need) in the subjects.

\section{RESULTS}

Our independent variable was protagonist appearance, and our dependent variable was the amount of empathy expressed per protagonist as measured via the empathy scale and earthquake question. Our data was not normally distributed (and also ordinal), therefore we used non-parametric statistical measures in our analysis.

40 male and 80 female subjects completed our survey. Their ages ranged from 18 - 76 years old (mean: 29.4, s.d. = 9.9). We did not find a significant impact on robot empathy ratings due to age or gender.

To analyze whether the amount of empathy expressed per protagonist related to robot appearance, we performed Friedman's Test to see whether there was a significant difference in the rankings. The rankings were: AUR: 1.95, Roomba: 2.18, Andrew: 3.21, Alicia: 3.65, and Anton: 4.01. $(\mathrm{p}<0.05)$. In answer to the earthquake question, respondents significantly favored Alicia (39\%) and Andrew $(47 \%)$ over AUR (6\%) and Roomba (8\%). Thus, people felt far more empathetic toward the humanoid robots, Alicia and Andrew, than to the mechanical-looking AUR and Roomba.

\section{DISCUSSION}

We found strong support for our hypothesis that people are more empathetic toward human-like robots and less empathetic toward mechanical-looking robots. This result is compatible with Simulation Theory which states that people mentally 'simulate' the situation of other agents in or- der to understand their mental and emotive state, and that the more similar the other agent is to the empathizer the stronger the empathy expressed. Our result also supports recent findings by Krach et al. who found that as the degree of anthropomorphization increases people neurologically view robots as being more like themselves [4].

Our findings contribute in several ways to the humanrobot interaction community. First, from a theoretical standpoint, our results inform the human likeness debate. It may be that when people feel uncomfortable around a humanbut-not-quite robot their simulation metric is upset.

Our second contribution is that designers of social robots now have another means by which to understand the acceptance of their robot. Before they even place a robot in front of a user, they are now aware of some of the possible biases their users might have in terms of their empathetic outlook. Since empathy is such a key component in effective social interaction, this result is something likely to be of interest.

Third, our results help to inform some recent robot ethics debates. Whitby raises a number of issues regarding the mistreatment of human-like robots. He argues that while people have the liberty to abuse property within the privacy of their own home, if their act of abuse causes harm to other human beings it is morally unacceptable [7]. Considering our results showed that people empathized nearly as much with the humanoid and android robot protagonists as they did with the human protagonist, witnessing human-like robot abuse could potentially bring emotional harm to other humans. On the other hand, our results also show that people probably wouldn't feel badly if they saw a mechanical looking housework robot being abused. So, in short, appearance does matter when it comes to designing ethical frameworks for robots, and this area warrants further investigation.

\section{ACKNOWLEDGMENTS}

This work is supported in part by the Qualcomm Research Studentship.

The authors would especially like to thank Guy Hoffman for permitting us the use of his AUR videos. We would also like to thank Pradipta Biswas, Ian Davies, Alison Merikangas, Stephen Murdoch, Marc Nobile, Rachel Schwartz, Graham Titmus, and Phil Tuddenham.

\section{REFERENCES}

[1] J. Goetz, S. Kiesler, and A. Powers. Matching robot appearance and behavior to tasks to improve human-robot cooperation. In IEEE ROMAN, 2003.

[2] P. J. Hinds, T. L. Roberts, and H. Jones. Whose job is it anyway? A study of human-robot interaction in a collaborative task. Hum.-Comp. Interact., 19(1), 2004.

[3] C.-C. Ho, K. F. MacDorman, and Z. A. Pramono. Human emotion and the uncanny valley: A GLM, MDS, and Isomap analysis of robot video ratings. In $A C M / I E E E H R I, 2008$.

[4] S. Krach, F. Hegel, B. Wrede, G. Sagerer, F. Binkofski, and T. Kircher. Can machines think? Interaction and perspective taking with robots investigated via fMRI. PLoS ONE, 3(7), July 2008.

[5] A. Meltzoff and R. Brooks. 'Like me' as a building block for understanding other minds: Bodily acts, attention, and intention. In F. Malle, L. Moses, and D. Baldwin, editors, Intention and Intentionality, pages 171-191. MIT Press, 2001.

[6] J. C. Turner. Social comparison, similarity and ingroup favouritism. In H. Tajfel, editor, Differentiation between socia groups: Studies in the social psychology of intergroup relations. Academic Press, 1978.

[7] B. Whitby. Sometimes it's hard to be a robot: A call for action on the ethics of abusing artificial agents. Interact. Comput., $20(3), 2008$ 